\title{
Stability-Indicating HPLC Method for the Determination of Cefcapene Pivoxil
}

\author{
Przemysław Zalewski • Judyta Cielecka-Piontek • \\ Piotr Garbacki · Anna Jelińska • \\ Marta Karaźniewicz-Łada
}

Received: 28 June 2012/Revised: 16 August 2012/ Accepted: 16 August 2012/Published online: 18 September 2012

(C) The Author(s) 2012. This article is published with open access at Springerlink.com

\begin{abstract}
The stability-indicating LC assay method was developed and validated for quantitative determination of cefcapene pivoxil in the presence of degradation products formed during forced degradation studies. An isocratic RPHPLC method was developed with a Lichrospher RP-18 $(250 \mathrm{~mm} \times 4.6 \mathrm{~mm}, 5 \mu \mathrm{m})$ column and the mobile phase composed of 45 volumes of acetonitrile and 55 volumes of mixture composed of citric acid $10 \mathrm{mmol} \mathrm{L}^{-1}$ and potassium chloride $18 \mathrm{mmol} \mathrm{L}^{-1}$. The flow rate of the mobile

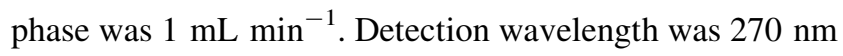
and temperature was $30^{\circ} \mathrm{C}$. Cefcapene pivoxil, similar to other cephalosporins, was subjected to stress conditions of degradation in aqueous solutions including hydrolysis, oxidation, and thermal degradation. The method was validated with regard to linearity, accuracy, precision, selectivity, and robustness. The method was applied successfully for the determination of cefcapene pivoxil during kinetic studies in aqueous solutions $(\mathrm{pH}$ and thermal degradation) and in solid state (oxidative, thermal, and radiolytic degradation).
\end{abstract}

Published in the special paper collection Advances in

Chromatography and Electrophoresis \& Chiranal 2012 with guest editor Jan Petr.

P. Zalewski $(\bowtie) \cdot$ J. Cielecka-Piontek · P. Garbacki ·

A. Jelińska

Department of Pharmaceutical Chemistry,

Faculty of Pharmacy, Poznan University

of Medical Sciences, Grunwaldzka 6, 60-780 Poznań, Poland

e-mail: pzalewski@ump.edu.pl

M. Karaźniewicz-Łada

Department of Physical Pharmacy and Pharmacokinetics,

Faculty of Pharmacy, Poznan University of Medical Sciences,

Grunwaldzka 6, 60-780 Poznań, Poland
Keywords Column liquid chromatography . Stability-indicating method $\cdot$ Cefcapene pivoxil

\section{Introduction}

Cefcapene pivoxil is a new, oral, third-generation cephalosporin. It has a broad spectrum of antibacterial activity against Gram-positive and Gram-negative bacteria, including Staphylococcus aureus [1]. Cefcapene pivoxil contains a carbamoyloxymethyl group at the $\mathrm{C} 3$ position, determining its antibacterial activity against Staphylococcus aureus. It was originally created by Shionogi \& Co., Ltd. and launched as Flomox in 1997. Previous studies proved that cephalosporins are susceptible to degradation in aqueous solutions [2-4] and in the solid state [5-9].

An essential parameter affecting the safety of drug use is chemical stability. The antibacterial activity of cephalosporins is attributed to their $\beta$-lactam moieties, which are very susceptible to chemical degradation. Most side effects of $\beta$-lactams are caused by their degradation products that hinder the development of analytical methods for the determination of cephalosporins. An optimal method is expected to separate and determine the substance to be examined in the presence of related products such as inprocess impurities, degradation products, and metabolites $[10,11]$. Although various methods are known for the determination of cefcapene pivoxil in biological fluids (blood, tissue, and cerebrospinal fluid) [12, 13], they are selective only for the metabolites of cefcapene pivoxyl. The current guidelines of the International Conference on Harmonization (ICH) require the development of stabilityindicating assay methods (SIAMs) suitable for the determination of drugs based on the analysis of stability test samples (Q1A-R2) (ICH Q2B, validation of analytical 
procedures, methodology). The stress tests are required to establish the effect of temperature and humidity in the solid state, the impact of temperature, light, oxidizing agent, $\mathrm{pH}$, buffer, and infusion fluids in solutions and the influence of biochemical processes on the formation of metabolites [14].

Microbiological methods have traditionally been used for determining cephalosporin. Yet, they are time-consuming and unable to differentiate antibiotics. HPLC methods, as opposed to microbiological techniques are rapid and specific. The aim of this work was to develop and validate an HPLC method with UV detection suitable for the identification, determination, and stability studies of cefcapene pivoxil.

\section{Experimental}

\section{Standards and Reagents}

Cefcapene pivoxil hydrochloride was obtained from Bepharm Ltd., 128 Xiangyin Road, Yangpu District, Shanghai, China. It is white or pale yellowish white crystalline powder slightly soluble in water.

All other chemicals and solvents were obtained from Merck KGaA (Germany) and were of analytical grade. High quality pure water was prepared using the Millipore purification system (Millipore, Molsheim, France, model Exil SA 67120).

\section{Instrumentation}

The analytical system consisted of a quaternary pump (L-7100), an autosampler (L-7200), a column oven (L-7360), and a diode array detector (L-7455) (all Merck Hitachi products). As the stationary phase, a Lichrospher RP-18 column, $5 \mu \mathrm{m}$ particle size, $250 \mathrm{~mm} \times 4 \mathrm{~mm}$ (Merck, Darmstadt, Germany) was used. The mobile phase composed of 45 volumes of acetonitrile and 55 volumes of mixture composed of citric acid $10 \mathrm{mmol} \mathrm{L}^{-1}$ and potassium chloride $18 \mathrm{mmol} \mathrm{L}^{-1}, \mathrm{pH}$ of the mobile phase was 2.36. The flow rate of the mobile phase was $1 \mathrm{~mL} \mathrm{~min}^{-1}$. The wavelength of the DAD detector was set at $270 \mathrm{~nm}$. Separation was performed at $30^{\circ} \mathrm{C}$.

\section{Procedure for Forced Degradation Study of Cefcapene Pivoxil}

\section{Degradation in Aqueous Solutions}

The degradation of cefcapene pivoxil in aqueous solutions was studied at $363 \mathrm{~K}$ in hydrochloric acid $\left(0.3 \mathrm{~mol} \mathrm{~L}^{-1}\right)$. The ionic strength of all solutions was adjusted to
$0.5 \mathrm{~mol} \mathrm{~L}^{-1}$ with a solution of sodium chloride $\left(4 \mathrm{~mol} \mathrm{~L}^{-1}\right)$. Degradation was initiated by dissolving an accurately weighed $10 \mathrm{mg}$ of cefcapene pivoxil in $50 \mathrm{~mL}$ of the solution equilibrated to $363 \mathrm{~K}$ in stopped flasks. At specified times, samples of the reaction solutions $(1 \mathrm{~mL})$ were instantly cooled with a mixture of ice and water.

\section{Oxidative Degradation}

Degradation was initiated by dissolving an accurately weighed $10 \mathrm{mg}$ of cefcapene pivoxil in $50 \mathrm{~mL}$ of $30 \%$ $\mathrm{H}_{2} \mathrm{O}_{2}$ solution equilibrated to $343 \mathrm{~K}$ in stopped flasks. At specified times, samples of the reaction solutions $(1 \mathrm{~mL})$ were instantly cooled with a mixture of ice and water.

Thermal Degradation

$5 \mathrm{mg}$ of samples of cefcapene pivoxil was weighed in $5 \mathrm{~mL}$ vials. In order to achieve the degradation of cefcapene pivoxil in solid state, their samples were immersed in heat chambers at 373 or $393 \mathrm{~K}$, both at $\mathrm{RH}=0 \%$. At specified time intervals, determined by the rate of degradation, the vials were removed, cooled to room temperature and their contents were dissolved in acetonitrile. The obtained solutions were quantitatively transferred into measuring flasks and diluted with acetonitrile to $25 \mathrm{~mL}$.

\section{Radiolytic Degradation}

$5 \mathrm{mg}$ of samples of cefcapene pivoxil was weighed in $5 \mathrm{~mL}$ vials and closed with a plastic stopper. The samples in the vials were exposed to irradiation in a linear electron accelerator LAE 13/9 (electron beam $9.96 \mathrm{MeV}$ and current intensity $6.2 \mu \mathrm{A}$ ) till they absorbed a dose of 25 and $400 \mathrm{kGy}$. The vials were removed and their contents were dissolved in acetonitrile. The obtained solutions were quantitatively transferred into measuring flasks and diluted with acetonitrile to $25 \mathrm{~mL}$.

\section{Results and Discussion}

Optimization of Chromatographic Conditions

In HPLC method with mobile phase composed of 70 volumes of mixture composed of potassium dihydrogen phosphate $1.56 \mathrm{~g} \mathrm{~L}^{-1}$, sodium dodecyl sulfate $1.22 \mathrm{~g} \mathrm{~L}^{-1}, 30$ volumes of acetonitrile and 10 volumes of methanol separation was unsatisfactory. In HPLC method with mobile phase composed of 40 volumes of acetonitrile and 60 volumes of mixture composed of citric acid $50 \mathrm{mmol} \mathrm{L}^{-1}$ and potassium chloride $30 \mathrm{mmol} \mathrm{L}^{-1}$ adjusted to $\mathrm{pH} 3$ by sodium hydroxide [12] peak asymmetry and resolutions were unsatisfactory. Change 


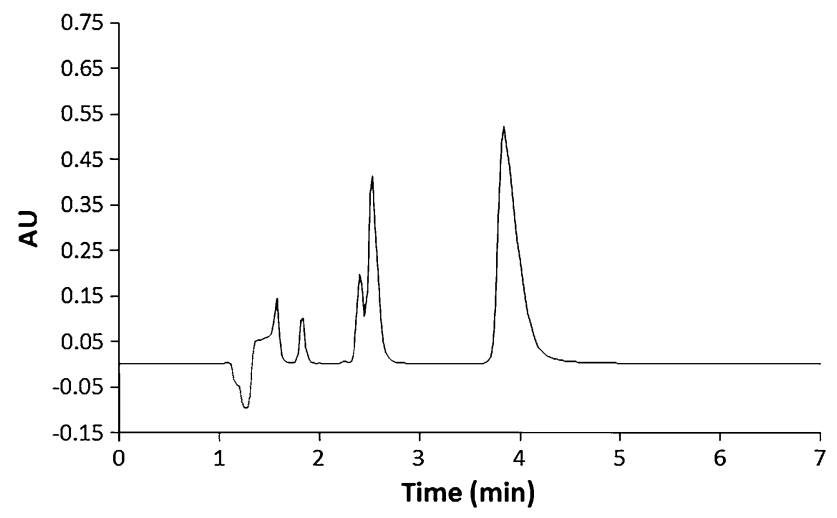

Fig. 1 The HPLC chromatogram of cefcapene pivoxil $\left(t_{\mathrm{R}}=3.84 \mathrm{~min}\right)$ in the presence of degradation products $\left(t_{\mathrm{R}}\right.$ from 1.57 to $2.53 \mathrm{~min}$ ) following incubation at $0.5 \mathrm{~mol} \mathrm{~L}-1$ HCl, at $363 \mathrm{~K}$ for $180 \mathrm{~min}$

to $\mathrm{pH} 2.4$ (without additions of sodium hydroxide) and in concentration of citric acid, potassium chloride and acetonitrile were satisfactory changing resolutions and peak asymmetry. It was observed that satisfactory resolution of cefcapene pivoxil (retention time $3.84 \mathrm{~min}$ ) and their degradation products (retention time from 1.57 to $2.53 \mathrm{~min}$ ) formed under various stress conditions was achieved when analysis of stressed samples was performed on an HPLC system using a C-18 column and a mobile phase composed of 45 volumes of acetonitrile and 55 volumes of mixture composed of citric acid $10 \mathrm{mmol} \mathrm{L}^{-1}$ and potassium chloride $18 \mathrm{mmol} \mathrm{L}^{-1}$. The detection was carried out at $270 \mathrm{~nm}$. The mobile phase flow rate was $1 \mathrm{~mL} \min ^{-1}$. Typical retention times of cefcapene pivoxil were about $3.84 \mathrm{~min}$ (Fig. 1). In blank sample, purity of peak of cefcapene pivoxil was $100 \%$ and peak asymmetry was 1.95 .

\section{Method Validation}

HPLC method was validated according to the International Conference on Harmonization Guidelines (ICH Q2B, validation of analytical procedures, methodology). The method was validated for parameters such as selectivity, linearity, precision, accuracy, and robustness.

\section{Selectivity}

The selectivity was examined for non-degraded and degraded samples [the solutions of cefcapene pivoxil after stress conditions of acid hydrolysis at $363 \mathrm{~K}$, oxidation $\left(\mathrm{H}_{2} \mathrm{O}_{2}\right)$, and thermal degradation $(373$ and $\left.393 \mathrm{~K})\right]$.

The HPLC method for determination of cefcapene pivoxil was found selective in the presence of degradation products as shown in Fig. 1. Peaks were symmetrical, clearly separated from each other (Fig. 1). Photodiode array detection
Table 1 Results of forced degradation studies

\begin{tabular}{lll}
\hline Stress conditions and time studies & $\begin{array}{l}\text { Degradation } \\
(\%)\end{array}$ & $\begin{array}{l}\text { Peak } \\
\text { purity }\end{array}$ \\
\hline $\begin{array}{l}\text { Acidic/0.5 mol L } \\
\quad 240 \mathrm{~min}\end{array}$ & 56.4 & 100.00 \\
$\begin{array}{l}\text { Oxidizing/30 \% H} \mathrm{H}_{2} / 343 \mathrm{~K} / \\
\quad 310 \mathrm{~min}\end{array}$ & 88.7 & 98.79 \\
Thermal/373 K/28 days & 9.4 & 100.00 \\
Thermal/393 K/28 days & 30.9 & 100.00 \\
Radiolytic/25 kGy & 1.7 & 99.98 \\
Radiolytic/400 kGy & 10.8 & 99.15 \\
\hline
\end{tabular}

${ }^{\text {a }}$ Peak purity values in the range of $98.5-100$ indicates a homogeneous peak

was used as an evidence of the specificity of the method and to evaluate the homogeneity of cefcapene pivoxil peaks. The peak purity values were more than $98.79 \%$ for cefcapene pivoxil at $270 \mathrm{~nm}$, what proves that degradants were not interfering with the mean peak (Table 1).

\section{Linearity}

Linearity was evaluated in the concentration range 20-240 $\mathrm{mg} \mathrm{L}^{-1}$ (10-120\% of the nominal concentration of cefcapene pivoxil during degradation studies). The samples of each solution were injected three times and each series comprised six experimental points.

The calibration plots were linear in the following concentration range $20-240 \mathrm{mg} \mathrm{L}^{-1}(n=6, r=0.9992)$. The calibration curve was described by the equation $y=a c$; $y=(5491604 \pm 239226) c$. The $b$ value, calculated from equation $y=\mathrm{a} c+b$, was insignificant because it was lower than the critical value $t_{\mathrm{b}}=b / S_{\mathrm{b}}$. Statistical analysis using Mandel's fitting test confirmed linearity of the calibration curves.

\section{Precision}

Precision of the assay was determined in relation to repeatability (intra-day) and intermediate precision (interday). In order to evaluate the repeatability of the methods, six samples were determined during the same day for three concentrations of cefcapene pivoxil. Intermediate precision was studied by comparing the assays performed on two different days.

The intra-day and inter-day precision values of measured concentration of cefcapene pivoxil, as calculated from linearity plots are given in Table 2 . The RSD values were 0.58 and $1.27 \%$, respectively, demonstrating that the method was precise.

Good recoveries were obtained for each concentration, confirming that the method was accurate (Table 2). 
Table 2 Intra-day, inter-day precision $(n=6)$ and recovery $(n=3)$ studies

\begin{tabular}{lll}
\hline $\begin{array}{l}\text { Spiked concentration } \\
\left(\mathrm{mg} \mathrm{L}^{-1}\right)\end{array}$ & $\begin{array}{l}\text { Measured concentration } \pm \mathrm{SD} \\
\left(\mathrm{mg} \mathrm{L}^{-1}\right)\end{array}$ & $\begin{array}{l}\text { RSD } \\
(\%)\end{array}$ \\
\hline Intra-day precision & & \\
160.0 & $160.46 \pm 0.78$ & 0.50 \\
200.0 & $200.21 \pm 1.13$ & 0.58 \\
240.0 & $240.79 \pm 1.09$ & 0.47 \\
Inter-day precision & & 1.27 \\
200.0 & $201.22 \pm 2.47$ & \\
Recovery studies & & 100.09 \\
$160.0(\sim 80 \%)$ & $160.15 \pm 0.65$ & 100.12 \\
$200.0(\sim 100 \%)$ & $200.25 \pm 1.03$ & 100.29 \\
$240.0(\sim 120 \%)$ & $240.69 \pm 1.41$ & \\
\hline
\end{tabular}

\section{Accuracy as Recovery Test}

The accuracy of the method was determined by recovering cefcapene pivoxil from the placebo. The recovery test was performed at three levels 80, 100, and $120 \%$ of the nominal concentration of cefcapene pivoxil during degradation studies. Three samples were prepared for each recovery level. The solutions were analyzed and the percentage of recoveries was calculated (Table 2).

\section{Limits of Detection (LOD) and Quantification (LOQ)}

The LOD and LOQ parameters were determined from the regression equation of cefcapene pivoxil: $\mathrm{LOD}=3.3 S_{y} / a$, $\mathrm{LOQ}=10 S_{y} / a$, where $S_{y}$ is a standard error and $a$ is the slope of the corresponding calibration curve.

Under applied chromatographic conditions, the LOD of cefcapene pivoxil was $4.24 \mathrm{mg} \mathrm{L}^{-1}$ and LOQ of cefcapene pivoxil was $12.85 \mathrm{mg} \mathrm{L}^{-1}$.

\section{Robustness}

The robustness of the procedure was evaluated after changing the following parameters: the composition of the mobile phase (concentration of acetonitrile in the range $43-47 \%$, concentration of citric acid in the range 9-11 $\mathrm{mmol} \mathrm{L}^{-1}$, concentration of potassium chloride in the range $16-20 \mathrm{mmol} \mathrm{L}^{-1}$ ), the $\mathrm{pH}$ of mobile phase in the range 2.34-2.38, the mobile phase flow rate (flow rate in the range $1.48-1.52 \mathrm{~mL} \mathrm{~min}^{-1}$ ), wavelength of absorption $(270 \pm 5 \mathrm{~nm})$, temperature $\left(30 \pm 2{ }^{\circ} \mathrm{C}\right)$. For each parameter change its influence on the retention time $\left(t_{\mathrm{R}}\right)$, resolution $\left(R_{\mathrm{S}}\right)$, area $(A)$ and asymmetry of peak was evaluated (Table 3). No significant changes in resolution, retention time, area and asymmetry of peak were observed and tested parameters were modified.
Table 3 Results of robustness studies

\begin{tabular}{|c|c|c|c|c|}
\hline Parameter & $t_{\mathrm{R}}$ & $R_{\mathrm{S}}^{\mathrm{a}}$ & $A$ & $\begin{array}{l}\text { Peak } \\
\text { asymmetry }^{\mathrm{b}}\end{array}$ \\
\hline Optimal & 4.27 & 1.87 & 3817826 & 1.95 \\
\hline $\mathrm{ACN}=47 \%$ & 4.32 & 1.75 & 3807469 & 1.85 \\
\hline $\mathrm{ACN}=43 \%$ & 4.32 & 1.92 & 3813561 & 1.98 \\
\hline Citric acid $=11 \mathrm{mmol} \mathrm{L}^{-1}$ & 3.95 & 1.64 & 3881498 & 1.87 \\
\hline Citric acid $=9 \mathrm{mmol} \mathrm{L}^{-1}$ & 3.95 & 1.53 & 3872026 & 1.69 \\
\hline $\begin{array}{l}\text { Potassium } \\
\text { chloride }=20 \mathrm{mmol} \mathrm{L}^{-1}\end{array}$ & 4.19 & 1.85 & 3798476 & 1.92 \\
\hline $\begin{array}{l}\text { Potassium } \\
\text { chloride }=16 \mathrm{mmol} \mathrm{L}^{-1}\end{array}$ & 4.16 & 1.75 & 3812361 & 1.88 \\
\hline pH 2.38 & 4.32 & 1.80 & 3700756 & 1.90 \\
\hline pH 2.34 & 4.32 & 1.83 & 3850258 & 1.92 \\
\hline$f=1.52 \mathrm{~mL} \mathrm{~min}^{-1}$ & 4.27 & 1.79 & 3766090 & 1.91 \\
\hline$f=1.48 \mathrm{~mL} \mathrm{~min}^{-1}$ & 4.37 & 1.75 & 3835079 & 1.89 \\
\hline$\lambda=275 \mathrm{~nm}$ & 4.32 & 1.86 & 3835709 & 1.94 \\
\hline$\lambda=265 \mathrm{~nm}$ & 4.32 & 1.86 & 3827682 & 1.95 \\
\hline$T=28^{\circ} \mathrm{C}$ & 4.32 & 1.87 & 3811839 & 1.95 \\
\hline$T=32{ }^{\circ} \mathrm{C}$ & 4.32 & 1.87 & 3810980 & 1.95 \\
\hline
\end{tabular}

${ }^{a}$ Peaks are separated to baseline if resolution is $>1.5$

${ }^{\mathrm{b}}$ Peak asymmetry $<1.50$ indicatives symmetry of peak

\section{Results of Forced Degradation Experiments}

During stability studies, a $20-80 \%$ degradation of the substance to be examined is to be achieved to qualify the assay method as able to indicate stability [14]. In previous studies, concerning the stability of cephalosporin reactions of acidic and basic hydrolysis occurred rapidly [4-8]. Cefcapene pivoxil was observed to be significantly degraded as a result of acidic hydrolysis and oxidative stress conditions at an increased temperature, although it was found to be relatively resistant to acidic hydrolysis at room temperature. After $240 \mathrm{~min}, 56.4 \%$ of cefcapene pivoxil degraded in $0.5 \mathrm{~mol} \mathrm{~L}^{-1} \mathrm{HCl}$, at $363 \mathrm{~K}$. Chromatograms of solutions obtained after degradation under acidic conditions are shown in Fig. 1. The main degradation products had retention times of about 1.57 and $2.53 \mathrm{~min}$. Since cefcapene pivoxil precipitated in alkaline solutions and it was impossible to estimate its degradation rate during basic hydrolysis. Cefcapene pivoxil proved to be fairly resistant to oxidative stress conditions. To accelerate degradation, an increased temperature was used. Cefcapene pivoxil showed desired stability in dry hot air (373 and $393 \mathrm{~K}$ after 28 days). The main degradation products had the same retention time as those observed during acidic hydrolysis and oxidative degradation. Cefcapene pivoxil was also resistant to radiolytic stress conditions where 1.66-10.84\% of it is degraded. The results of forced degradation experiments on cefcapene pivoxil under various stress conditions are summarized in Table 1. 


\section{Conclusions}

The isocratic RP-LC method developed for the analysis of cefcapene pivoxil in their pharmaceutical preparations is selective, precise, and accurate. The method is useful for routine analysis due to short run time. This method can be used for determining the stability of cefcapene pivoxil in its pharmaceutical preparations.

Open Access This article is distributed under the terms of the Creative Commons Attribution License which permits any use, distribution, and reproduction in any medium, provided the original author(s) and the source are credited.

\section{References}

1. Koga T, Rikimaru T, Tokunaga N, Higashi T, Nakamura M, Ichikawa Y, Matsuo K (2011) J Infect Chemother 17(4):499-503

2. Ikeda Y, Ban J, Ishikawa T, Hashiguchi S, Urayama S, Horibe H (2008) Chem Pharm Bull 56(10):1406-1411
3. Jelińska A, Dobrowolski L, Oszczapowicz I (2004) J Pharm Biomed Anal 35(5):1273-1277

4. Sugioka T, Asano T, Chikaraishi Y, Suzuki E, Sano A, Kuriki T, Shirotsuka M, Saito K (1990) Chem Pharm Bull 38(7): 1998-2002

5. Medenecka B, Jelińska A, Zając M, Bałdyka M, Juszkiewicz K, Oszczapowicz I (2009) Acta Pol Pharm 66(5):563-569

6. Jelińska A, Medenecka B, Zając M, Knajsiak M (2008) Acta Pol Pharm 65(2):261-265

7. Zając M, Jelińska A, Zalewski P (2005) Acta Pol Pharm 62(2):89-94

8. Jelińska A, Dudzińska I, Zając M, Oszczapowicz I, Krzewski W (2005) Acta Pol Pharm 62(3):183-187

9. Zając M, Jelińska A, Dobrowolski L, Oszczapowicz I (2003) J Pharm Biomed Anal 32(6):1181-1187

10. Cielecka-Piontek J, Michalska K, Zalewski P, Jelińska A (2011) Curr Pharm Anal 7(4):213-227

11. Zalewski P, Cielecka-Piontek J, Jelińska A (2012) Cent Eur J Chem 10(1):121-126

12. He X, Sugawara M, Takekuma Y, Miyazaki K (2004) Antimicrob Agents Chemother 48(7):2604-2609

13. Fujimoto M (2001) Int J Antimicrob Agents 18(5):489-494

14. Sehrawat R, Maithani M, Singh R (2010) Chromatographia 72(1-2):1-6 